\title{
Water column tomographic inversion with a network of drifting buoys
}

S.M. Jesus sjesus@ualg.pt

C. Soares csoares@ualg.pt

N.E. Martins nmartins@ualg.pt

Institute for Systems and Robotics, Universidade do Algarve,

Campus de Gambelas, PT-8005-139 Faro, Portugal.

Ref.: invited to Acoustics'08, Paris, France, June 2008.

\begin{abstract}
The estimation of ocean environmental properties by means of the inversion of acoustic signals has in several occasions been performed using a single vertical array of acoustic receivers, with a towed acoustic source as an attempt to ensure a rapid spatial coverage of the area of interest, as only a single ocean transect is "seen" at each time.

Ideally, one would like to obtain an instantaneous picture of the complete area (volume) under observation. However, the resulting acoustic observations, hence environmental estimates, are not simultaneous in time. Using multiple acoustic receiving arrays appears to be a natural step towards both increasing the spatial coverage, and obtaining simultaneous environmental estimates of different ocean transects. It also gives a higher chance to capture spatial transient features, as for example solitons. Using multiple receiver arrays represents the addition of a new spatial dimension at the receiving end and opens up the number of possibilities to a Nx2D or full 3D view of the ocean. Taking support on the data set of the RADAR'07 experiment (July 9 - 16, 2007) where data was simultaneously collected on three vertical arrays, this paper explores space coherent processing of the several receiving arrays and Nx2D or 3D environmental constrained water coloumn matched-field inversion.
\end{abstract}

Acknowledgement: this work was funded by FCT, Portugal under programs POCI, POSI and POCTI. 